\title{
Epigenetic dysregulation and poorer prognosis in DAXX-deficient pancreatic neuroendocrine tumours
}

\section{Dear Editor,}

Exome sequencing of sporadic pancreatic neuroendocrine tumours (PNETs) has identified mutually exclusive mutations in the chromatin regulators $\alpha$-thalassaemia/mental retardation X-linked (ATRX) and death-associated protein 6 (DAXX) genes in $43 \%$ of cases (18 and $23 \%$ of cases respectively in 68 cases studied) (Elsässer et al. 2011, Jiao et al. 2011). ATRX and DAXX are chromatin remodellers; their loss leads to alternative lengthening of telomeres (ALT) and chromosomal instability (CIN) (Heaphy et al. 2011). ALT is a telomerase-independent mechanism for the maintenance of telomere stabilisation. Although it was initially reported that $A T R X / D A X X$ mutant tumours had superior 10-year survival and outcome (Jiao et al. 2011), a recent larger study on 243 tumours demonstrated that ATRX and DAXX loss and associated ALT in PNETs correlates with CIN, advanced tumour stage, the development of metastases and poorer progression-free survival (PFS) and overall survival (OS) (Marinoni et al. 2014).

ATRX interacts with DNA methyltransferases 3A and 3L (DNMT3A/3L), known as ATRX-DNMT3A-DNMT3L (ADD) (Hashimoto et al. 2010). DNMT3A and its accessory protein, DNMT3L, contain a histone H3 lysine 4 (H3K4) methyl-interacting ADD domain which links DNA methylation with unmodified $\mathrm{H} 3 \mathrm{~K} 4$. This interaction is one of the three described protein domains that provide a functional link between DNA methylation and histone modification. These interactions are pivotal for maintaining accurate replication of histone methylation patterns in newly replicated chromatin and in the subsequent fidelity of gene expression. ATRX interacts directly with DAXX, which functions as a chaperone for the deposition of the histone variant $\mathrm{H} 3.3$ at repeat sequences across the genome, including CpG islands and telomeric, pericentric and ribosomal repeats (Clynes et al. 2013). DAXX is a highly specific histone chaperone that discriminates H3.3 from other $\mathrm{H} 3$ variants. Mutually exclusive mutations in $A T R X$ and $D A X X$ are also found in neurological tumours, including neuroblastomas, paediatric glioblastomas, oligodendrogliomas and medulloblastomas (Clynes et al. 2013). Notably, H3.3 is mutated in paediatric glioblastoma and bone tumours, and H3.3 mutations are often associated with changes in global DNA methylation (Salomoni 2013, Maze et al. 2014).

Because of the known interaction between ATRX and DNMT3A/3L and the interplay between ATRX and DAXX, it is likely that PNETs with a loss of these tumour suppressor genes would have different genome-wide DNA methylation patterns as compared to those tumours that retain this function.

In this study, we sought to determine the genome-wide DNA methylation and copy number aberration profiles in ATRX/DAXX-positive and ATRX/DAXXnegative PNETs using the Infinium 450K HumanMethylation BeadArray (Illumina Inc., San Diego, CA, USA).

Only ATRX/DAXX-positive tumours and tumours with a loss of either ATRX or DAXX were considered. Included in the study were 12 age-matched, normal control pancreatic tissue samples (endocrine and exocrine) because of the extreme rarity of isolated pancreatic islet cell samples. Cases that showed heterogeneous ATRX or DAXX loss were also excluded so as to avoid difficulties in drawing firm conclusions.

In total, 53 formalin-fixed paraffin-embedded tumour specimens (46 primaries and seven liver metastases from 39 cases) were included in this study (Fig. 1A). Of these, 27 specimens (51\%) had lost either ATRX $(n=9$; $33 \%)$ or DAXX ( $n=18 ; 67 \%)$ protein expression, as determined by immunohistochemistry (Anti-ATRX (SAB4502258) and Anti-DAXX (HPA008736) antibodies (both rabbit polyclonal) were provided by Sigma-Aldrich). Endothelial cells that stained positive for ATRX and DAXX served as the internal control in each immunochemistry section from cases that lacked expression of the corresponding protein. Seven of 26 (27\%) low-grade primary tumours (G1, ki-67 of <2\%) exhibited ATRX/DAXX loss as compared to 13 of 19 (68\%) intermediate-grade tumours

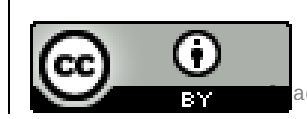




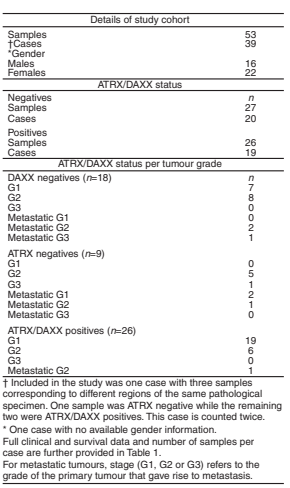

B

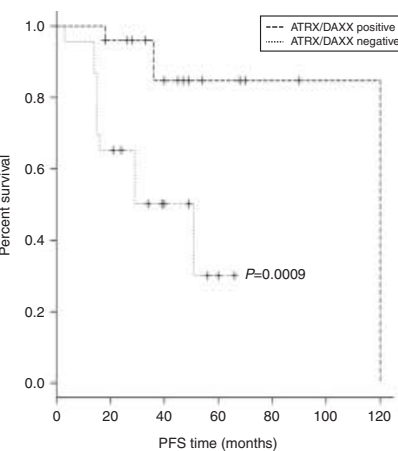

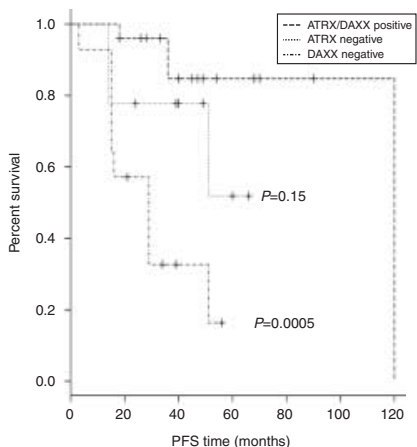

D

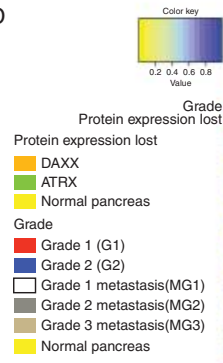

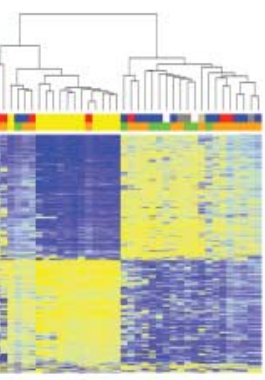

E

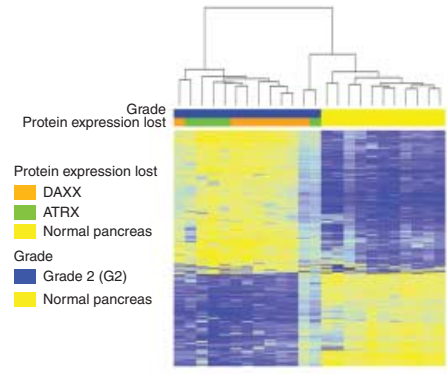

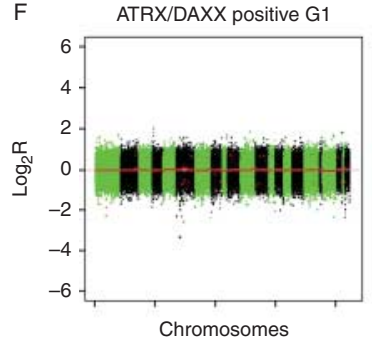

Chromosomes

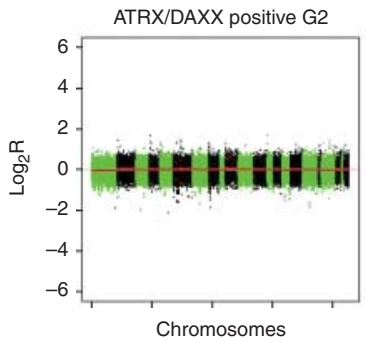

ATRX/DAXX negative G1

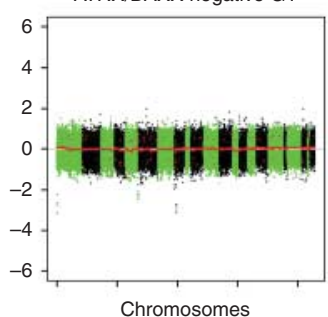

Chromosomes

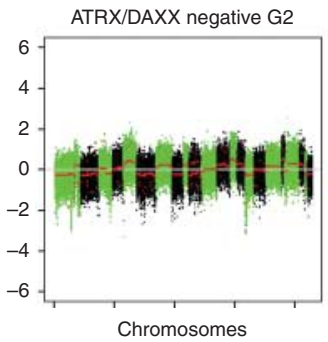

ATRX/DAXX negative G1 met

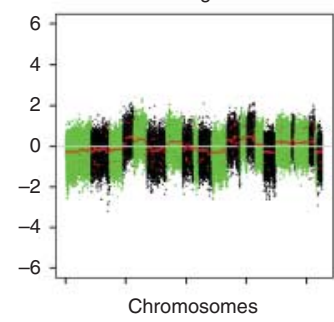

Chromosomes

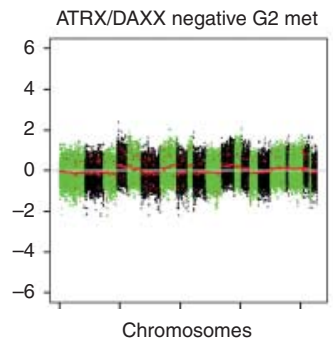

\section{Figure 1}

(A) Details of the study cohort. (B and C) Kaplan-Meier survival curves for ATRX- and DAXX-negative cases $(n=17)$ as compared to ATRX/DAXXpositive cases $(n=17)$ and ATRX-negative $(n=8)$ and DAXX-negative $(n=9)$ cases analysed independently and compared to ATRX/DAXX-positives cases respectively. The 5 -year PFS was $85 \%$ for positive cases, $52 \%$ for ATRXnegative cases and $16 \%$ for DAXX-negative cases. (D) Unsupervised cluster analysis using the top 1000 MVPs that showed segregation of ATRXnegative (in green) and DAXX-negative (in orange) tumours as compared to normal pancreatic tissue. Of the six ATRX/DAXX-negative tumour samples (from six cases) that clustered with the normal control pancreatic samples, only one case (G1, DAXX-negative) progressed (PFS 51 months), and two cases had no follow-up data. Of the six metastatic samples included in this study, two of them (both DAXX-negative) had a matched G1 tumour sample. One of them grouped tightly with the G1 primary tumour. DNA methylation values ( $\beta$-value $0-1)$ are represented using a colour scale, where yellow $=$ low methylation and blue $=$ high methylation. Samples are shown on the $x$-axis, and probes are shown on the $y$-axis. (E) Confirmation of tumour segregation primarily by grade and then by ATRX or DAXX status using the top 1000 MVPs. (F) Copy number variation (CNV) profiles associated with low and intermediate PNETs. The top panel demonstrates increasing CNV across the genome for low-grade (G1, ki-67 <2\%) tumours, with the least CNV occurring in the ATRX/DAXXpositive primary (left). Increasing CNV alterations are seen in the ATRX/DAXX-negative tumour (middle) and most CNV alterations occur in the ATRX/DAXX-negative G1 liver metastasis (right). The same is observed in intermediate grade (G2, ki-67 3-20\%) tumours in the bottom panel. Segmented copy numbers are shown as a red line, and sequential chromosomes are shown in green and black (chr. 1-22).

Published by Bioscientifica Ltd. 
(G2, ki-67 2-20\%) ( $P=0.008$, Fisher's exact test). One G3 and six of seven metastatic specimens also exhibited ATRX/DAXX loss.

Survival analysis was based on 34 cases with available follow-up data (eight ATRX-negative, nine DAXX-negative, and 17 ATRX/DAXX-positive cases; Table 1). Data were not available for two DAXX-negative and one ATRX/DAXX-positive case. Case 37 (counted twice), which had both ATRX-negative and ATRX/DAXXpositive samples, was also excluded. Analysis of the ATRX/DAXX-negative cases demonstrated that eight cases (five DAXX-negative and three ATRX-negative cases) progressed within 5 years of follow-up, whereas nine cases (four DAXX-negative and five ATRX-negative cases) did not. The majority of positive cases (13 of 17) remained progression-free over the period of study. Poorer 5-year PFS was observed in ATRX-negative and DAXXnegative cases as compared to ATRX/DAXX-positive cases $(P=0.0009)$ (Fig. 1B). When ATRX-negative and DAXXnegative cases were analysed independently, a loss of DAXX led to a significantly poorer PFS at 5 years (DAXX loss: $16 \%, P=0.0005$; ATRX loss: $52 \%, P=0.15$; and no loss 85\% 5-year PFS; Fig. 1C). All $P$ values were obtained using a Cox regression model.

Depending on the different pair-wise comparisons, certain samples that had been assigned to unique arrays had to be removed to allow for the correction of the batchto-batch variation which occurs when running the Illumina 450K HumMeth array. Comparison between all ATRX/DAXX-negative and ATRX/DAXX-positive tumours identified 58 methylation-variable positions (MVPs). Independent comparisons of either DAXX-negative $(n=18)$ or ATRX-negative $(n=7)$ with ATRX/DAXX-positive tumours $(n=23)$ revealed 4352 MVPs and 34 differentially methylated regions (DMRs) and 258 MVPs and one DMR respectively. When ATRX-negative and DAXX-negative tumours were compared, we identified 196195 MVPs and 6708 DMRs. Taken together, these observations demonstrate that genome-wide DNAm changes are associated with a loss of DAXX expression and not a loss of ATRX. A Benjamini-Hochberg $(\mathrm{BH})$ adjusted $P$ value of $<0.05$ that corrected for multiple testing (false discovery rate) was used throughout the various comparisons in order to identify significantly methylated variable positions.

When ATRX-negative and DAXX-negative tumours were compared as one group to normal pancreatic samples, we identified 133938 MVPs (BH adjusted $P$ value $<0.05)$ and 4664 DMRs. A heat map that plotted the top 1000 MVPs for this comparison demonstrated that tumours were separated primarily by grade and then by ATRX or DAXX status (Fig. 1D). This was further confirmed by the unsupervised clustering of G2 tumours (eight DAXX-negative and five ATRX-negative cases) alone against normal pancreatic control samples for the top 1000 MVPs (Fig. 1E).

Of the 26 ATRX/DAXX-negative tumours (Fig. 1D), 20 samples $(77 \%)$ were grouped into a distinct cluster. However, six ATRX/DAXX-negative tumour samples had intermediate methylation profiles and clustered with the normal control pancreatic samples (Fig. 1D). Of them, five were DAXX-negative (four G1 and one G2 tumours), and one was ATRX-negative (a G2 tumour). When comparing G2 tumours vs normal pancreas, there were 127683 MVPs (adjusted $P$ value $<0.05$ ) and 4337 DMRs. However, for G1 vs normal pancreas, 31480 MVPs and 300 DMRs were identified. This indicates that low-grade (G1) tumours and normal pancreas have similar methylation profiles.

Copy number variation (CNV) was determined in ATRX/DAXX-negative and ATRX/DAXX-positive tumours using DNA methylation data as previously described (Feber et al. 2014). ATRX-negative and DAXX-negative tumours demonstrated increased $\mathrm{CNV}$ as compared to positive tumours (Fig. 1F). The genome-wide CNV rate was quantified by determining the cumulative size of genomic alterations (bp) in genomic regions that harboured a copy number change of $<-0.3$ (for loss) or $>0.3$ (for gain). Across the 23 ATRX/DAXX-positive primary tumours, there were $1.4 \times 10^{9}$ bp of $\mathrm{CNV}$ in total (range per tumour $250000-4.3 \times 10^{6}$, median $1.6 \times 10^{6}$ ). Across the 25 ATRX/ DAXX-negative primary tumours, there were $2.4 \times 10^{9} \mathrm{bp}$ of $\mathrm{CNV}$ in total (range per tumour $279000-8.0 \times 10^{7}$, median $1.5 \times 10^{7}$ ).

This is the first study of genome-wide DNA methylation in ATRX/DAXX-positive and ATRX/DAXX-negative PNETs. The finding of a higher incidence of ATRX and DAXX loss in intermediate-grade (G2) tumours may account for the worse PFS and OS previously observed (Marinoni et al. 2014); however, this finding requires validation in a separate clinical cohort.

Genome-wide DNA methylation analysis identified significantly more MVPs in DAXX-negative vs ATRX/ DAXX-positive tumours as compared to ATRX-negatives vs ATRX/DAXX-positive tumours (4352 and 258 respectively). This suggests that DAXX deficiency drives genome-wide methylation changes, potentially through the functional loss of H3.3 deposition (which binds DAXX in a highly specific manner and loads it into DNA, whereas ATRX is a co-factor that is involved in DAXX targeting chromatin) and also through binding with the maintenance DNA methyl transferase DNMT1 (Salomoni 2013). Mutations in H3.3

Published by Bioscientifica Ltd. 
Table 1 Clinical information and survival data

\begin{tabular}{|c|c|c|c|c|c|c|c|c|c|}
\hline $\begin{array}{l}\text { Patient } \\
\text { ID }\end{array}$ & Sample ID & Age & Gender & $\begin{array}{l}\text { Primaryl } \\
\text { metastasis }\end{array}$ & Grade & $\begin{array}{l}\text { Protein } \\
\text { expression } \\
\text { lost }\end{array}$ & $\begin{array}{c}\text { Total } \\
\text { follow-up } \\
\text { (months) }\end{array}$ & PFS months & OS months \\
\hline \multicolumn{10}{|c|}{ ATRX/DAXX-negative tumours } \\
\hline 1 & 1B2T_MG2_N & 46 & $M$ & Metastasis & MG2 & ATRX & 54 & 51 & 54 \\
\hline 3 & 3A28T2_G3_N & 53 & $M$ & Primary & G3 & ATRX & 83 & 14 & 83 \\
\hline 4 & 4A4T_G2_N & 60 & $M$ & Primary & $\mathrm{G} 2$ & DAXX & 85 & 3 & 85 \\
\hline 7 & 7C3T_MG1_N & 81 & $\mathrm{~F}$ & Metastasis & MG1 & ATRX & 60 & No progression & Alive \\
\hline \multirow[t]{3}{*}{8} & 8A2T_G1_N & 49 & $\mathrm{~F}$ & Primary & G1 & DAXX & NA & NA & NA \\
\hline & 8A3T1_G1_N & & & & G1 & DAXX & & & \\
\hline & 8A3T2_G1_N & & & & G1 & DAXX & & & \\
\hline 11 & 11A1T_MG3_N & 56 & M & Metastasis & MG3 & DAXX & 20 & 16 & 20 \\
\hline \multirow[t]{3}{*}{12} & 12A3T1_MG2_N & 62 & $\mathrm{~F}$ & Metastasis & MG2 & DAXX & 106 & 29 & Alive \\
\hline & 12A9T2_MG2_N & & & Metastasis & MG2 & DAXX & & & \\
\hline & 12A10T_G2_N & & & Primary & G2 & DAXX & & & \\
\hline \multirow[t]{4}{*}{13} & 13A12T_G2_N & 73 & $M$ & Primary & G2 & DAXX & 55 & 15 & Alive \\
\hline & 13A13T_G2_N & & & & $\mathrm{G} 2$ & DAXX & & & \\
\hline & 13A14T_G2_N & & & & G2 & DAXX & & & \\
\hline & 13A19T_G2_N & & & & G2 & DAXX & & & \\
\hline 15 & 15A6T_G1_N & 36 & M & Primary & G1 & DAXX & 39 & No progression & Alive \\
\hline 19 & 19A7T_G1_N & 30 & $\mathrm{~F}$ & Primary & G1 & DAXX & 54 & 51 & 54 \\
\hline 21 & 21A4T_G2_N & 66 & M & Primary & $\mathrm{G} 2$ & ATRX & 39 & No progression & Alive \\
\hline 25 & 25A3T_G1_N & 72 & $\mathrm{~F}$ & Primary & G1 & DAXX & 34 & No progression & Alive \\
\hline 26 & 26A2T_G2_N & 21 & $M$ & Primary & G2 & DAXX & 21 & No progression & Alive \\
\hline 30 & 30C6T_G2_N & 54 & M & Primary & G2 & ATRX & 40 & No progression & Alive \\
\hline 31 & 31A4T_G2_N & 74 & $\mathrm{~F}$ & Primary & G2 & ATRX & 24 & No progression & Alive \\
\hline 34 & 34A10T_G2_N & 69 & $F$ & Primary & G2 & DAXX & NA & NA & NA \\
\hline 35 & 35A7T_G1_N & 23 & $F$ & Primary & G1 & DAXX & 56 & No progression & Alive \\
\hline 36 & 36A3T_G2_N & 57 & M & Primary & G2 & ATRX & 72 & 14 & 72 \\
\hline 37 & 37A3T1_G2_N & 40 & $\mathrm{~F}$ & Primary & G2 & ATRX & 49 & No progression & Alive \\
\hline 38 & 38A4T_MG1_N & 84 & $\mathrm{~F}$ & Metastasis & MG1 & ATRX & 66 & No progression & Alive \\
\hline \multicolumn{10}{|c|}{ ATRX/DAXX-positive tumours } \\
\hline 2 & 2B2T_G1_P & 67 & M & Primary & G1 & No Loss & 90 & No progression & Alive \\
\hline 5 & 5B5T2_G1_P & 60 & M & Primary & G1 & No Loss & 84 & 18 & Alive \\
\hline \multirow[t]{4}{*}{6} & 6A6T_G1_P & 51 & $\mathrm{~F}$ & Primary & G1 & No Loss & 68 & No progression & Alive \\
\hline & 6A8T1_G1_P & & & & G1 & No Loss & & & \\
\hline & 6A8T2_G1_P & & & & G1 & No Loss & & & \\
\hline & 6A8T3_G1_P & & & & G1 & No Loss & & & \\
\hline 9 & 9A9T2_G1_P & 62 & $\mathrm{~F}$ & Primary & G1 & No Loss & 70 & No progression & Alive \\
\hline 10 & 10A7T_G1_P & 45 & $F$ & Primary & G1 & No Loss & 54 & No progression & Alive \\
\hline 14 & 14B8T_G1_P & NA & NA & Primary & G1 & No Loss & 45 & No progression & Alive \\
\hline \multirow[t]{2}{*}{17} & 17A2T_G1_P & 53 & $\mathrm{~F}$ & Primary & G1 & No Loss & 47 & No progression & Alive \\
\hline & 17A4T_G1_P & & & & G1 & No Loss & & & \\
\hline \multirow[t]{2}{*}{18} & 18A4T_G1_P & 64 & $\mathrm{~F}$ & Primary & G1 & No Loss & 40 & No progression & Alive \\
\hline & 18A5T_G1_P & & & & G1 & No Loss & & & \\
\hline 20 & 20A3T_MG2_P & 69 & $\mathrm{~F}$ & Metastasis & MG2 & No Loss & NA & NA & NA \\
\hline 22 & 22A6T_G1_P & 72 & $M$ & Primary & G1 & No Loss & 18 & No progression & Alive \\
\hline \multirow[t]{2}{*}{23} & 23D2T_G1_P & 54 & $\mathrm{~F}$ & Primary & G1 & No Loss & 28 & No progression & Alive \\
\hline & 23D7T_G1_P & & & & G1 & No Loss & & & \\
\hline 24 & 24A6T2_G2_P & 47 & $\mathrm{~F}$ & Primary & G2 & No Loss & 84 & 36 & Alive \\
\hline 27 & 27A6T_G2_P & 58 & $M$ & Primary & $\mathrm{G} 2$ & No Loss & 33 & No progression & Alive \\
\hline 28 & 28A8T_G2_P & 66 & $\mathrm{~F}$ & Primary & $\mathrm{G} 2$ & No Loss & 18 & No progression & Alive \\
\hline 29 & 29C4T_G1_P & 71 & $\mathrm{~F}$ & Primary & G1 & No Loss & 26 & No progression & Alive \\
\hline 32 & 32B4T_G1_P & 26 & $M$ & Primary & G1 & No Loss & 126 & 120 & Alive \\
\hline 33 & 33E13T_G1_P & 58 & $\mathrm{M}$ & Primary & G1 & No Loss & 26 & No progression & Alive \\
\hline \multirow[t]{2}{*}{37} & 37A4T_G2_P & 40 & $\mathrm{~F}$ & Primary & G2 & No Loss & 49 & No progression & Alive \\
\hline & 37A5T_G2_P & & & & G2 & No Loss & & & \\
\hline 39 & 39IIFT_G2_P & 58 & $\mathrm{~F}$ & Primary & G2 & No Loss & 162 & 36 & Alive \\
\hline
\end{tabular}

PFS, progression-free survival; OS, overall survival; NA, not available. The three samples from case 37 are different regions of the same surgical pathological specimen. However, because of intra-tumoral heterogeneity, we observed that one sample was ATRX-negative, whereas the remaining two were ATRX/DAXX-positive. For this reason, case 37 was not considered in the survival analysis.

http://erc.endocrinology-journals.org DOI: 10.1530/ERC-15-0108
(C) 2015 The authors Printed in Great Britain
Published by Bioscientifica Ltd 
occur commonly in paediatric brain tumours (Maze et al. 2014) and are known to lead genome-wide methylation changes. Because ATRX interacts with the de novo DNA methyl transferases DNMT3A/3L, its impact on genomewide methylation in ATRX-deficient PNETs is less marked.

In addition to investigating genome-wide DNA methylation changes, $\mathrm{CNV}$ was determined from the non-normalised methylated and unmethylated signal intensities of the $450 \mathrm{~K}$ array probes as previously described (Feber et al. 2014). In keeping with previously reported data in PNETs (Marinoni et al. 2014) and other solid tumours, we found a higher incidence of CNV alterations in tumours that had lost ATRX and DAXX (Heaphy et al. 2011). We also demonstrated that ATRX/DAXX-negative tumours have a poorer PFS as compared to ATRX/DAXX-positive cases (30 vs $85 \%, P=0.0009$ ) and that DAXX-negative tumours have the poorest 5 -year PFS overall $(16 \%, P=0.0005)$.

We demonstrated that even though they have mutually exclusive mutations, ATRX-and DAXX-deficient PNETs have distinct genome-wide DNA methylation profiles. Loss of DAXX and not ATRX appears to be the driver event in altering genome-wide methylation changes in PNETs. Previous studies have analysed PNETs with ATRX and DAXX loss in a single cohort, because mutations in these genes are predominantly mutually exclusive. These findings are also relevant to other neurological tumours which are driven by ATRX and DAXX loss.

Finally, we demonstrated that DAXX-negative tumours have the poorest 5-year PFS, and we therefore suggest more aggressive disease course. However, further validation of these findings is warranted in a separate clinical cohort.

$$
\begin{aligned}
& \text { Christodoulos P Pipinikas }{ }^{1,{ }^{*}} \\
& \text { Harpreet Dibra }{ }^{1, *} \\
& \text { Anna Karpathakis }{ }^{1} \\
& \text { Andrew Feber }{ }^{1} \\
& \text { Marco Novelli }{ }^{2} \\
& \text { Dahmane Oukrif }{ }^{2} \\
& \text { Guiseppe Fusai }{ }^{3} \\
& \text { Roberto Valente }{ }^{3} \\
& \text { Martyn Caplin }{ }^{4} \\
& \text { Tim Meyer }{ }^{1,4} \\
& \text { Andrew Teschendorff }{ }^{1,5} \\
& \text { Christopher Bell }{ }^{1} \\
& \text { Tiffany J Morris }{ }^{1} \\
& \text { Paolo Salomoni }{ }^{1} \\
& \text { Tu-Vinh Luong }{ }^{6} \\
& \text { Brian Davidson }{ }^{3} \\
& \text { Stephan Beck }{ }^{1} \\
& \text { Christina Thirlwell }{ }^{1,4}
\end{aligned}
$$

${ }^{1}$ Medical Genomics Laboratory, University College London Cancer Institute, University College London, 72 Huntley Street, London WC1E 6BT, UK

${ }^{2}$ Department of Pathology, University College London, London, UK

${ }^{3}$ Department of Surgery, Royal Free Hospital, London, UK

${ }^{4}$ Royal Free Hospital NET Unit, London, UK

${ }^{5}$ CAS-MPG Partner Institute for Computational Biology, Shanghai Institute for Biological Sciences, Shanghai 200031, China

${ }^{6}$ Department of Pathology, Royal Free Hospital, London, UK

*(C P Pipinikas and H Dibra contributed equally to this work)

(Correspondence should be addressed to C Thirlwell; email: christina.thirlwell@ucl.ac.uk)

Declaration of interest

The authors declare that there is no conflict of interest that could be perceived as prejudicing the impartiality of the research reported.

\section{Funding}

C Thirlwell, C P Pipinikas and A Karpathakis were supported by Cancer Research UK. The Raymond and Beverly Sackler Foundation funded the NET Biobank UCL. D Oukrif was supported by the Experimental Cancer Medicine Centre. The UCH/UCL Biomedical Research Centre funded T Meyer. The Brain Tumour Charity, European Research Council and National Institute of Health Research funded P Salomoni. The Beck lab was supported by the Wellcome Trust (grant number 99148), a Royal Society Wolfson Research Merit Award (number WM100023) and EU-FP7 projects EPIGENESYS (number 257082), IDEAL (number 259679) and BLUEPRINT (number 282510).

Author contribution statement

H Dibra, M Caplin, T Meyer, P Salomoni, S Beck and C Thirlwell conceived and designed the study. C P Pipinikas, H Dibra, A Karpathakis, A Feber, M Novelli, D Oukrif, G Fusai, R Valente, A Teschendorff, C Bell, T J Morris, T-V Luong, B Davidson and C Thirlwell performed the data acquisition, analysis and interpretation. C P Pipinikas, H Dibra and C Thirlwell drafted the manuscript. C P Pipinikas, A Karpathakis, A Feber, M Novelli, M Caplin, T Meyer, A Teschendorff, C Bell, P Salomoni, S Beck and C Thirlwell critically revised the manuscript. C P Pipinikas, A Karpathakis, D Oukrif, T J Morris and T-V Luong provided technical support.

\section{Acknowledgements}

All patients included in the study were recruited through the Royal Free Hospital Neuroendocrine Unit. Informed consent was obtained from all patients before they entered the study. All samples were fully anonymised. Study approval was obtained from a local Research Ethics Committee (REC reference number 09/H0722/27).

Published by Bioscientifica Ltd 


\section{References}

Clynes D, Higgs DR \& Gibbons RJ 2013 The chromatin remodeller ATRX: a repeat offender in human disease. Trends in Biochemical Sciences 38 461-466. (doi:10.1016/j.tibs.2013.06.011)

Elsässer SJ, Allis CD \& Lewis PW 2011 Cancer. New epigenetic drivers of cancers. Science 331 1145-1146. (doi:10.1126/science.1203280)

Feber A, Guilhamon P, Lechner M, Fenton T, Wilson GA, Thirlwell C, Morris TJ, Flanagan AM, Teschendorff AE, Kelly JD et al. 2014 Using high-density DNA methylation arrays to profile copy number alterations. Genome Biology 15 R30. (doi:10.1186/gb-2014-15-2-r30)

Hashimoto H, Vertino PM \& Cheng X 2010 Molecular coupling of DNA methylation and histone methylation. Epigenomics 2 657-669. (doi:10.2217/epi.10.44)

Heaphy CM, de Wilde RF, Jiao Y, Klein AP, Edil BH, Shi C, Bettegowda C, Rodriguez FJ, Eberhart CG, Hebbar S et al. 2011 Altered telomeres in tumors with ATRX and DAXX mutations. Science 333425. (doi:10.1126/science.1207313)

Jiao Y, Shi C, Edil BH, de Wilde RF, Klimstra DS, Maitra A, Schulick RD, Tang LH, Wolfgang CL, Choti MA et al. 2011 DAXX/ATRX, MEN1, and mTOR pathway genes are frequently altered in pancreatic neuroendocrine tumors. Science 331 1199-1203. (doi:10.1126/science.1200609)

Marinoni I, Kurrer AS, Vassella E, Dettmer M, Rudolph T, Banz V, Hunger F, Pasquinelli S, Speel E-J \& Perren A 2014 Loss of DAXX and ATRX are associated with chromosome instability and reduced survival of patients with pancreatic neuroendocrine tumors. Gastroenterology 146 453-60.e5. (doi:10.1053/j.gastro.2013.10.020)

Maze I, Noh K-M, Soshnev AA \& Allis CD 2014 Every amino acid matters: essential contributions of histone variants to mammalian development and disease. Nature Reviews. Genetics 15 259-271. (doi:10.1038/nrg3673)

Salomoni P 2013 The PML-interacting protein DAXX: histone loading gets into the picture. Frontiers in Oncology 3 152. (doi:10.3389/fonc. 2013.00152)

Received in final form 15 April 2015

Accepted 21 April 2015

Made available online as an Accepted Preprint

21 April 2015
Published by Bioscientifica Ltd. 